\title{
$\underline{\text { La relégation des récidivistes : enjeux politique et pénal }}$
}

La loi du 27 mai 1885, dite loi sur la relégation des récidivistes, représente une date importante dans l'histoire pénale française. Cette loi introduit effectivement en droit pénal le principe de dangerosité et aménage une présomption irréfragable d'incorrigibilité censée mesurer le degré de dangerosité d'un criminel ou d'un délinquant récidiviste. Loi d'élimination sociale comme la décline ses promoteurs, la relégation s'articule comme une peine secondaire qui s'ajoute à une peine principale et se traduit par une mesure de sûreté et d'éloignement prise contre des condamnés jugés « incorrigibles ». La relégation va ainsi permettre à une puissance coloniale de se débarrasser de 1887 à 1938 de près de 22163 condamnés : 4270 relégués en Nouvelle-Calédonie (1887-1897) et 17375 en Guyane (1887-1938). Les cibles de cette loi sont des délinquants récidivistes condamnés pour des motifs de vol simple ou de vagabondage aggravé et la relégation est essentiellement prononcée par des tribunaux correctionnels. Pour se prononcer, le magistrat est simplement tenu de comptabiliser le nombre de condamnations inscrites au casier judiciaire d'un prévenu ou d'un condamné et si ce nombre emporte une des combinaisons prévues par le quantum de la loi, il est obligé à l'issue de son verdict de prononcer la relégation.

Ce résultat, aussi surprenant qu'il puisse paraitre, est issu de la volonté d'un homme, Léon Gambetta et de ses successeurs opportunistes au Parlement tout au long d'un débat qui mobilise députés et sénateurs durant près de deux ans (1883-1885). Cette loi, si sévère soit-elle, s'inscrit au sein d'une réforme pénale qui tient compte des principales conclusions dégagés par des experts du crimes et des peines à partir de la seconde moitié du XIXème siècle et s'articule autour d'une nouvelle lecture de la criminalité qui fait désormais autorité à partir de 1880. Cette dernière se décline en deux chapitres auxquels il faut adapter la réponse répressive afin de conjurer au mieux les chiffres délivrés par la statistique judiciaire qui ne cessent depuis 1825 d'indiquer une hausse alarmante du nombre de délits produits en récidive. La relégation correspond ainsi au premier volet d'une réforme pénale qui envisage désormais les criminels et les délinquants en deux entités distinctes : les criminels et les délinquants de profession ou incorrigibles, ceux contre qui l'emprisonnement est totalement inutile et qui manifestent par la réitération de leurs crimes et de leurs délits leur incorrigibilité avérée, leur témébilité spéciale, et les criminels et les délinquants d'accident, ceux qui n'en sont qu'à leur première infraction et qu'il faut coûte que coûte préserver du contact corrupteur des prisons. Le dispositif mis en place à partir de 1881 contre la récidive par les républicains opportunistes consacre ainsi l'échec de l'option pénitentiaire et lui substitue une réponse originale qui s'articule autour d'une mesure d'élimination, la relégation promulguée le 27 mai 1885 , et d'une mesure de prévention, la loi sur la libération conditionnelle promulguée le 14 
août 1885 et renforcée le 26 mars 1891 par la loi sur le sursis à exécution de la peine.

Comprendre la relégation nécessite de saisir les différents enjeux qui mobilisent ses promoteurs et imposent de découpler son analyse autour de deux approches : politique et pénale. Ces différents niveaux d'analyse permettent ainsi de répondre tour à tour à différentes questions que posent d'emblée cette loi. Tout d'abord, pourquoi un personnel politique qui de 1881 à 1885 s'emploie à mettre en œuvre un ensemble de grandes libertés démocratiques et qui assure une école libre, laïque et obligatoire vote en parallèle le 12 mai 1885 une loi aussi sévère que celle de la relégation ? Pour comprendre l'enjeu politique que représente cette loi, il importe en premier lieu de l'envisager au sein du projet politique d'envergure aménagé par les républicains opportunistes à partir de 1880. Dans cette lecture, la relégation correspond à une mesure de régulation sociale qui vise à préserver un modèle républicain présenté tout à la fois comme un régime de libertés publiques mais également comme un régime d'ordre. Les délinquants et les vagabonds récidivistes, à la suite de l'épisode de la Commune, représentent une menace contre les biens et les propriétés mais également contre l'ordre social où ils sont associés en permanence à la figure de l'insurgé toujours prête à faire basculer les couches populaires dans la voie de la révolte et du crime. La relégation permet tout à la fois de séparer les récidivistes de la communauté nationale et de présenter le personnel politique républicain comme le mieux à même pour assurer la protection des citoyens.

Néanmoins, cette loi se saisit également de délinquants et de criminels que son principal défenseur sur la scène parlementaire, le ministre de l'intérieur Pierre Waldeck-Rousseau, ne cesse de décliner comme des «incorrigibles », c'est-à-dire comme des individus que la pénalité classique n'est plus en mesure de corriger ou d'amender. Pour saisir l'enjeu que représente cette loi au niveau pénal, il est nécessaire de questionner le concept de délinquants et de criminels dits « incorrigibles » que la relégation consacre en droit à partir de 1885. Le processus d'élaboration de cette loi repose sur un modèle original caractéristique du mode d'action des républicains opportunistes en matière pénale et s'appuie sur de nombreux acteurs extérieurs à la sphère parlementaire qui composent autant d'espaces d'expertise qui viennent épauler le personnel politique et l'orienter dans sa décision. Les républicains opportunistes enregistrent ainsi à travers cette loi les doléances de nombreux experts du crime et des peines qui réclament l'instauration d'une loi contre les actes commis par des récidivistes dits «incorrigibles». Ces espaces d'expertises s'étendent de la sphère des criminologues, à celle des statisticiens et des magistrats qui chacun dans leur domaine d'activité respectif ont dégagé ou constaté l'existence de criminels et de délinquants dits «incorrigibles » et réclament une mesure d'exclusion outre-mer pour s'assurer d'eux. Les républicains opportunistes, en charge d'assurer l'ordre public, héritent donc d'un problème, celui posé par la récidive et qui 
constitue depuis l'épisode de la Commune le signe le plus caractéristique du déclin moral et politique de la nation. Aiguillés par des savoirs positifs qui ont permis de faire émerger une nouvelle lecture de la récidive, ces derniers aménagent une réforme pénale d'urgence visant à pallier aux défaillances du code pénal de 1810 et visant à répondre à un problème qui connaît un retentissement conséquent au sein de l'opinion publique, celui posé par les récidivistes incorrigibles.

\section{$\underline{\text { A. Éloigner des indésirables et sauvegarder la communauté nationale }}$}

Les promoteurs de la relégation sur la scène législative poursuivent un triple objectif : dénoncés par la droite d'être le parti exclusif des libertés, la relégation permet aux républicains opportunistes de prouver qu'ils sont également les représentants du parti de l'ordre. Il en va ainsi de leur crédibilité et le gouvernement de Jules Ferry doit démontrer en instaurant la relégation qu'il sait se montrer ferme sur la question sécuritaire :

«Ce sont des questions qui sont, on peut le dire, à l'ordre du jour des pays européens ; le mal n'est pas seulement contagieux, il est épidémique; et il me semble que s'il est un gouvernement qui ait le devoir strict de s'en préoccuper, s'il est une société qui doive chercher les moyens d'y mettre un frein, de prévenir, de réprimer la récidive, c'est le gouvernement républicain, ce sont les sociétés démocratiques1. »

Ce faisant, cette loi permet en parallèle au personnel politique opportuniste de se décliner comme le protecteur des plus modestes en proie aux attaques de délinquants et de criminels récidivistes :

«Oui, c'est dans les couches populaires qu'on réclame avec plus d'ardeur la transportation des récidivistes, parce que c'est là qu'on souffre le plus de cette plaie sociale. Ce ne sont pas les fils de la bourgeoisie, comme le disait M. le rapporteur, qui en souffre le plus, ce sont les fils de travailleurs, ceux qui vivent dans un contact forcé avec ces parvenus de la police correctionnelle et du crime, et qui souffrent de la flétrissure que leur inflige ce contact odieux2. »

A travers le vote de cette loi, les républicains opportunistes peuvent ainsi démontrer aux

1 Dreyfus F., Annales de la Chambre des députés, Imprimerie du Journal Officiel, Paris, séance du 21 avril 1883, JO du 22 avril 1883 , p. 28.

2 Waldeck-Rousseau P., Ibid., séance du 26 avril 1883, JO du 27 avril 1883, p. 120. 
électeurs des classes populaires qu'ils cherchent à rallier qu'ils sont en mesure de les défendre et de les protéger. Mais ce positionnement permet également aux partisans de la relégation d'écarter d'une main l'accusation de leurs challengers intransigeants. Emmenée à l'Assemblée nationale par Georges Clemenceau, l'opposition intransigeante ne cesse de dénoncer cette loi comme une arme destinée à frapper les plus pauvres et réclame la mise en œuvre d'une réforme sociale et pénitentiaire de grande ampleur seule à même selon elle d'enrayer la courbe de la récidive. A l'inverse, les républicains opportunistes présentent cette loi comme un préalable indispensable avant la mise en place et l'arrivée des bénéfices des différentes mesures sociales et économiques qu'ils souhaitent impulser. La relégation est donc une loi de sauvegarde républicaine qui s'intègre dans un projet politique d'ensemble et qui accompagne le processus d'intégration des couches populaires mis en place à partir de 1880 par les républicains opportunistes :

«On parle d'améliorer les conditions sociales dans lesquelles tant de déshérités doivent vivre, mais il n'est pas une amélioration qui s'impose avec plus d'urgence que l'assainissement des milieux où ils se trouvent, où ils travaillent, où ils souffrent 3 !...»

Débarrasser la société de l'élément incorrigible est un objectif qui présente plusieurs garanties pour les républicains opportunistes. Le projet politique porté par ces derniers a pour objectif de créer une communauté politique en associant les couches populaires aux destinées de la nation grâce à l'octroi du suffrage universel masculin et de droits économiques et sociaux. Cette nouvelle communauté associe les anciennes «classes laborieuses/classes dangereuses » à l'exercice politique. Craint et écarté du pouvoir tout au long du XIXème siècle, les notables des différents régimes n'ont eu de cesse de regarder le peuple comme profondément «immoral » et par là dangereux. Car c'est de l'immoralité selon eux que provient toute la misère du peuple et c'est son manque d'éducation et l'absence de propriété qui le maintient dans la pauvreté. Les républicains portent un regard différent sur les ouvriers et les paysans et cherchent à édifier un pacte sur la base duquel les couches populaires deviendraient détentrices en propre de la destinée commune du pays. Il s'agit d'associer à la souveraineté comme puissance publique détenue initialement par l'État, la souveraineté du peuple qui possède, grâce au suffrage universel masculin, le pouvoir de légitimation de la puissance publique assuré et garanti par la Constitution. Le droit de vote permet ainsi de lancer une passerelle entre le peuple et ses dirigeants et pacifie les affrontements politiques grâce au jeu démocratique. La citoyenneté se présente ainsi comme un mode d'incorporation des classes populaires à la régulation politique. L'éducation des classes populaires grâce à une école

3 Waldeck-Rousseau P., Ibid., p. 121. 
libre, laïque et obligatoire couplée à l'octroi de droits économiques et sociaux permet de former les futurs citoyens et d'en faire des électeurs rationnels. Mais ce droit de vote, fondement du nouveau pacte social, présente un risque du fait de son caractère universel et il faut préserver les électeurs des classes populaires du contact «corrupteur» que sont susceptibles de faire peser sur eux les récidivistes :

«Oui ! messieurs, dans un pays de suffrage universel, dans une nation où comme dans la nôtre tous les citoyens ont une part égale dans l'exercice de la citoyenneté, il importe au plus haut degré de prendre des mesures ayant pour but de prévenir l'augmentation de la pénalité, pourquoi ? Parce qu'il importe d'épurer la source même de tous les pouvoirs, la nation de ferments impurs, parce qu'il importe de combattre, de détruire, si faire ce peut, ces germes qui se répandent dans toutes les parties du corps social pour y engendrer le crime et le vice4. »

Selon Pierre Waldeck-Rousseau, la relégation est une loi de «sauvegarde »sociale, c'est-à-dire une loi qui dans l'esprit du ministre de l'intérieur vise à protéger d'abord et avant tout des citoyens nouvellement acquis au régime. Alors que la République cherche à rassembler, le récidiviste ne cesse par son exemple de «corrompre » la jeunesse et les classes populaires et de les amener sur la pente du crime et du délit. Afin d'empêcher « cette contagion, cette inoculation du vice », il faut envisager la relégation comme une loi d'urgence nationale visant à protéger « la partie saine de la population » contre des éléments «corrupteurs ». Le risque majeur que font peser les récidivistes provient essentiellement de leur exemple «dépravant» qui risque de « contaminer» les classes populaires, celles qui vivent en contact étroit avec eux. La relégation permet ainsi de désolidariser le lumpen récidiviste des couches laborieuses nouvellement acquises au régime et d'éviter que ce dernier ne les «contaminent». Car c'est au sein du peuple que «l'armée » des récidivistes recrute et se démultiplie presque mathématiquement. La relégation agit ainsi comme un limes et établit une véritable frontière afin de sauvegarder le projet politique porté par les républicains opportunistes. On ne peut saisir le schéma de pensée du ministre de l'intérieur si l'on ne tient pas compte de sa vision organiciste du corps social et de la prégnance des catégories intellectuelles « d'imitation sociale » et de «milieu » qui font le lit de sa réflexion. Les récidivistes vivent dans le même milieu que les classes populaires et, à partir de ce milieu, ils peuvent «contaminer » tous les autres membres y évoluant. L'expulsion des récidivistes est donc un préalable indispensable avant d'envisager et d'autoriser toute réforme sociale au sein des classes populaires, avant que la

4 Dreyfus F., Ibid., séance du 21 avril 1883, JO du 22 avril 1883, p. 28. 
République ne puisse enfin agir là où elle le souhaite.

La relégation est donc une mesure préventive à prendre «vis-à-vis de ces éléments extérieurs, de ces agents de corruption qui exercent sur les jeunes générations des classes laborieuses » une «influence néfaste et délétère. » Elle entend ainsi s'ériger en rempart pour protéger les premières victimes des récidivistes, les enfants et les adolescents issus des classes populaires et elle accompagne et préserve tout à la fois la mise en place de mesures visant à secourir et à éduquer les enfants qui sont prises à la même époque par les gouvernements républicains.

Reléguer les récidivistes pour séparer de leur «contact corrupteur» les classes populaires, permet en premier lieu au ministre de l'intérieur de se présenter comme le seul à défendre les plus modestes contre leurs agissements, comme le seul à porter secours aux classes populaires qui subissent leurs agressions. Mais c'est également garantir aux gouvernants que ce même peuple cesse de produire des «ennemis» de l'intérieur en stoppant la «corruption» que les récidivistes opèrent en son sein. Ici, le peuple reste toujours motif d'inquiétude pour les dirigeants et les mesures prises par les républicains opportunistes pour assister et protéger les enfants des ouvriers signalent cette appréhension. Car c'est essentiellement du peuple que provient la menace. Peuple qu'il est grand temps d'éduquer, de pacifier et d'associer à l'exercice de la souveraineté nationale. La relégation participe ainsi à l'effort de moralisation des couches populaires qui de la lutte contre l'alcoolisme à la lutte contre la pornographie accompagne le processus d'intégration républicain. Et en procédant ainsi, la relégation exclut physiquement mais également politiquement des individus qui n'ont pas la capacité à devenir des électeurs car l'exercice de ce droit souverain réclame des précautions et ne peut être confié à n'importe qui. Aux côtés des femmes, des malades mentaux, des mineurs et des étrangers, le criminel est exclu de la citoyenneté en étant écarté du droit de vote. Se mettant en dehors du pacte républicain, en dérogeant au contrat social, le criminel signifie la frontière entre le «bon grain » de «l'ivraie » populaire, il est la figure-limite qui permet de circonscrire très précisément celui qui a la capacité pour exercer les droits garantis par sa citoyenneté de celui qui ne l'a pas. Les relégués, ennemis de l'intérieur, impossibles citoyens, sont donc bannis d'un territoire où ils n'ont pas leur place et où ils représentent une menace pour l'ensemble du corps social.

\section{B. L'émergence de l'homme dangereux}

Le code pénal, dans la conception héritée des Conventionnels qui l'ont rédigé en 1791, doit assurer l'ordre public, c'est-à-dire la conciliation entre la liberté et la sûreté des citoyens. La 
protection de l'ordre public résulte d'un contrat passé entre l'État et les citoyens et traduit la garantie assurée entre les devoirs et les droits des gouvernés et des gouvernants. La sûreté de l'espace privé, c'est-à-dire des biens et des personnes, vient ainsi directement compléter celle de l'espace public. L'ordre public correspond donc à la garantie d'un espace social sécurisé où chacun peut jouir de ses biens et de ses déplacements en toute quiétude. Les républicains opportunistes ont pour mission à leur tour d'assurer l'ordre public et s'inscrivent dans la filiation des principes hérités par la Révolution française. Le pénal représente un véritable enjeu politique pour ces derniers et agit comme un outil de régulation sociale destiné à ordonner la société afin de l'organiser dans le sens des valeurs qu'ils défendent. Et cette adaptation du droit pénal permet en parallèle de répondre aux nombreux bouleversements qui secouent la société française à partir de 1880. L'essor de l'industrialisation, l'émergence d'un prolétariat urbain, l'accroissement de l'exode rural, la multiplication des déplacements grâce à l'extension du réseau ferré entraînent une angoisse liée à la rupture des liens communautaires traditionnels et au basculement de la France dans l'ère industrielle. Angoisse qui est également alimentée par la Grande dépression économique qui sévit en France au moment où la loi sur la relégation est en déb at aux chambres. Impulser l'ordre et la sécurité est donc nécessaire pour organiser une société en pleine mutation mais est également nécessaire pour garantir la protection des biens et des propriétés contre la menace que peuvent représenter tous les exclus que génère ce nouvel ordre économique. Dans ce schéma, les récidivistes se heurtent constamment aux valeurs bourgeoises défendues et revendiquées par la République que sont le travail et la propriété.

Comme l'ont démontré Georges Rusche et Otto Kirchheimer, les conditions économiques propres à toutes sociétés données ont une influence sur leur système pénal. La relégation, en frappant essentiellement des vagabonds et des délinquants récidivistes, les exclue également d'un marché du travail où ils n'ont pas leur place et permet à des colonies destinées à les recevoir de jouer à plein le rôle de «soupapes sociales» de leur métropole. La relégation répond ainsi à différentes préoccupations et s'insère dans un projet qui vise à préserver l'ordre sous un triple aspect : social, économique et politique. Et le bras armé de cette préservation repose en partie sur le réaménagement du système pénal auquel s'attellent les républicains opportunistes dès leur arrivée au pouvoir.

La relégation consacre ainsi l'échec du code pénal de 1810 et à travers lui, l'échec du système pénitentiaire. En dénonçant l'échec global de la prison, les députés opportunistes s'en prennent essentiellement à la législation du code pénal de 1810 qui a selon eux consacré la prison comme peine principale. La relégation est donc une mesure d'urgence visant à compléter le code pénal tout en attendant son éventuelle réforme prévue par un décret du 26 mars 1887 . La 
relégation permet de réformer un système pénal qui semble n'être plus suffisamment efficace pour réprimer la criminalité et venir à bout d'une récidive dont les chiffres statistiques ne cessent d'accuser une hausse exponentielle. La récidive est une obsession pour les professionnels du droit et pour le personnel politique en charge de la politique criminelle et du contrôle social. Elle constitue le moteur de la réforme juridique et suscite des interrogations sur la validité de la peine et, au-delà, sur la nature humaine et sur sa réceptivité à la correction. Elle est également une question centrale à l'aune de laquelle les pénalistes peuvent apprécier l'efficacité de tout système pénal donné. Ainsi, lorsque la récidive croît, le système pénal prouve son insuffisance et il est alors nécessaire de le réformer. La relégation vient ainsi en appoint du code pénal de 1810 et, même si elle ne le contrarie pas totalement, le complète. Car le code ne tient pas compte de la nouvelle dichotomie qui fait autorité à la fin du XIXème siècle entre les criminels d'accident et ceux d'habitude. Le code pénal de 1810 mélange effectivement des primo-délinquants et des délinquants récidivistes en les condamnant tous deux à la prison. Et l'accroissement de la récidive et l'introduction de la relégation signalent ici l'échec de cette institution qui, loin de corriger tous ceux qu'on lui abandonne, ne fait que reconduire le crime et la délinquance. Car en prison le «novice» est vite initié par le «cheval de retour » et ne manque pas dans ce schéma de récidiver à sa libération. En faisant de la prison la peine générale censée amender tous ceux qu'on lui abandonne, le code pénal a une part de responsabilité dans la recrudescence des chiffres de la récidive.

A partir de 1872, la plupart des experts de la pénalité et du crime considèrent que le droit pénal doit se décliner en deux chapitres : un consacré aux primo-délinquants ou criminels d'occasion et un autre consacré aux délinquants d'habitude ou criminels incorrigibles. A l'homme criminel doté d'un libre arbitre du début du siècle succède une dichotomie entre criminels d'habitude et criminels d'accident sur laquelle va s'articuler une réforme pénale dont la relégation n'est qu'un volet.

Ce partage est le fruit de la conjonction de plusieurs facteurs qui accompagnent la réflexion sur le récidivisme de 1832 à 1885 . Il a tout d'abord sa source dans le mouvement d'individualisation des peines, amorcé avec la loi du 28 avril 1832 sur les circonstances atténuantes, et qui conduit à porter l'intérêt et l'action de la justice non plus sur le seul acte isolé de l'infracteur mais sur l'infracteur lui-même. Ce mouvement qui tend de plus en plus à individualiser la peine au criminel est renforcé par de nouvelles techniques d'identification comme le casier judiciaire ou plus tardivement l'anthropométrie qui permettent aux juges de mieux connaître les antécédents judiciaires des récidivistes et donc de mieux adapter la sanction au regard de la réitération de l'acte criminel. Ces techniques d'identification sont essentielles pour assister le magistrat dans son verdict et lui permettent d'adapter la peine en fonction du caractère récidivant ou non du condamné. 
L'étatisation des techniques d'identification et un meilleur maillage policier et judiciaire du territoire permettent en parallèle d'alimenter un facteur fondamental dans l'émergence du récidiviste incorrigible, la statistique judiciaire. Le criminel incorrigible que la relégation consacre en 1885 est d'abord et avant tout une catégorie dégagée par la statistique criminelle qui va l'isoler et lui donner une existence officielle à travers l'enregistrement continu des pratiques des magistrats au sein du Compte général de l'administration de la justice criminelle en France. Alors que les crimes de sang ne cessent de chuter, la statistique judiciaire met en exergue un accroissement continu de la récidive correctionnelle qui va peu à peu donner l'impression qu'un groupe de délinquants semble totalement insensible à la pénalité classique. La prison ne paraît effectivement plus d'aucun secours pour s'assurer d'eux et il semble pour les commentateurs du Compte qu'ils aient fait du vol et du vagabondage une véritable «profession ». Cette existence, marquée par une récidive qui les conduit à de courts séjours en prison, consacre leur irréductibilité à la pénalité classique et par là leur incorrigibilité avérée.

Ces chiffres servent également de matériau aux travaux conduits par les criminologues et par les anthropologues du crime qui valident à leur tour l'existence de criminels incorrigibles et qui réclament de nouvelles mesures pour les réprimer efficacement. Érigeant le crime en objet scientifique, Cesare Lombroso affirme en 1876 dans son ouvrage L'uomo delinquente l'existence de criminels et de criminels incorrigibles dont il faut débarrasser la société et les transporter dans des colonies suffisamment éloignées des métropoles où ils n'ont pas leur place. En France, Alexandre Lacassagne se montre également partisan de la transportation des criminels et des délinquants récidivistes incorrigibles. Vivant à rebours de tout progrès social, incapables de profiter des bienfaits offerts par une société trop avancée pour eux, les « incorrigibles » représentent un danger sur le sol de la métropole et doivent être transportés dans un milieu suffisamment «vierge » afin de pouvoir y recommencer leur vie.

Les criminologues et les statisticiens ne sont pas les seuls à réclamer une loi permettant de s'assurer de criminels et de délinquants incorrigibles et dangereux. Les magistrats, et ce dès 1872 , réclament également leur expulsion. Au lendemain de la répression de la Commune, l'Assemblée nationale vote le 25 mars 1872 sur la proposition du vicomte d'Haussonville une loi instaurant une commission d'enquête sur le régime des établissements pénitentiaires. Après la défaite et la débâcle contre la Prusse, le sentiment commun est une aspiration à la restauration d'un « ordre moral». L'épisode de la Commune a pointé du doigt les vices cachés de la société comme la misère, l'ignorance, l'enfance abandonnée mais également la criminalité et le vagabondage. La question pénitentiaire apparaît donc avec une urgence criante, largement négligée par un Second Empire regardé comme le principal responsable du déclin national. Le problème principal qui résume à lui 
seul l'état de la question pénitentiaire lorsqu'elle est saisie par la Chambre à cette date est celui de la récidive. La récidive est le symptôme qui traduit assez fidèlement le fait que la France est « dans une situation morale inférieure à celle des autres peuples ». La statistique judiciaire juge et sonne le glas du système pénitentiaire français : il ne parvient pas à «moraliser » ceux qu'on lui confie et doit donc être entièrement réformé. Les maisons d'arrêt et de justice et les maisons centrales sont considérées comme les principales sources de «démoralisation » des criminels et, par là, comme les principales responsables de la récidive. Mais à la réforme des prisons départementales qui aboutit au vote d'une loi sur l'encellulement le 5 juin 1875, d'Haussonville préconise la mise en place d'une «libération préparatoire », c'est-à-dire d'une libération conditionnelle associée à des sociétés de patronages. Mais une réforme en profondeur des maisons départementales ne suffit pas, il faut également en soustraire les « criminels incorrigibles ». Reprenant à son compte la dichotomie entre délinquants d'habitude et délinquants d'occasion, d'Haussonville souhaite que cette distinction constitue désormais « le fond de la science pénitentiaire » qui doit dorénavant empêcher un criminel d'accident de devenir un criminel d'habitude. Il propose ainsi de créer une peine de transportation contre les récidivistes afin de «purger » les prisons de l'élément « récidivistes, surveillés ou non, chez lesquels les tribunaux auraient reconnu des penchants vicieux incorrigibles ».

Cette initiative reçoit de plus le soutien du personnel judiciaire qui la sollicite également. Soucieuse de recueillir leurs avis au sujet de la réforme pénitentiaire, la commission d'enquête d'Haussonville fait parvenir à chaque cours d'appel ainsi qu'à la Cour de cassation un questionnaire à l'intérieur duquel figure l'opportunité de transporter ou non les récidivistes. A l'exception notable du procureur de la cour d'appel de Paris, les vingt-sept cours d'appel et la Cour de Cassation se prononcent toutes unanimement pour la transportation des récidivistes incorrigibles :

«Les récidivistes incorrigibles forment une armée toujours prête pour le désordre et la guerre civile : on en a fait une cruelle expérience lors de l'insurrection de la Commune de Paris. Il en sera ainsi dans toutes les occasions : le récidiviste, déshabitué du travail, aigri par le séjour des prisons, devient l'ennemi irréconciliable de l'ordre social ${ }^{5}$. »

A la suite de la commission d'Haussonville, la récidive connaît un véritable engouement et concentre l'attention de nombreux experts du crime et des peines. En mars 1878, la Société générale des prisons lance une enquête internationale sur la récidive et interroge différentes autorités publiques européennes en charge de la question pénale sur l'opportunité de transporter les

5 Cour d'appel de Poitiers, Enquête parlementaire sur le régime des établissements pénitentiaires, Imprimerie Nationale, Paris, 1874, t. V, p. 492. 
« récidivistes incorrigibles ». L'activité de la Société générale des prisons, créée en 1877, et du Conseil supérieur des prisons, créé en 1875, autour de la question de la récidive témoigne de la volonté de refonte du système pénitentiaire à travers toutes les questions suggérées par son accroissement. L'incidence de ces «espace[s] d'expertise » sur la politique pénale républicaine au début de la IIIème République a été analysée en détail par Martine Kaluszynski. La Société Générale des Prisons, créée par un arrêté du 22 mai 1877, a pour but de contribuer à l'amélioration du régime pénitentiaire en France. A travers son organe, le Bulletin de la Société Générale des Prisons, s'expriment de nombreux juristes et des spécialistes de la question pénale. Dès sa première séance, le 27 juin 1877, la Société générale des prisons aborde immédiatement l'étude du thème de la récidive et certains de ses membres, parmi lesquels le conseiller à la Cour de cassation et membre du Conseil supérieur des prisons Charles Petit, proposent la transportation des récidivistes. Alors que le débat sur l'opportunité de déporter les récidivistes bat son plein à la Société générale des prisons, le vicomte d'Haussonville, membre de cette société et du Conseil supérieur des prisons, propose à ce dernier l'examen d'une proposition tendant à établir une transportation perpétuelle contre les récidivistes. La commission du Conseil adapte cette proposition et propose de ne transporter que les récidivistes réputés les plus « dangereux », c'est-à-dire ceux condamnés pour des délits qui indiquent chez eux la présence d'instincts « pervers ». Cette proposition de loi est ensuite soumise au ministre de l'intérieur qui décide de ne pas y donner suite. Néanmoins, l'activité déployée par ces experts de la pénalité en faveur de l'enregistrement de la transportation des récidivistes au niveau législatif donne un écho conséquent à cette mesure et la présente comme le recours le mieux à même pour contrarier un «problème », celui posé par les récidivistes incorrigibles. L'initiative de ces agents censés être les plus au fait de la question pénale constitue ainsi un préalable important et a une incidence déterminante sur le programme de réformes pénales souhaité par les républicains opportunistes et qui vont arriver aux affaires moins de deux ans plus tard.

Les criminels et les délinquants dangereux, définis par de nouveaux savoirs et désignés au sein de l'espace public par de nouveaux outils de mesure, représentent une double menace : ils nuisent aux biens et aux propriétés et correspondent à une menace d'ordre politique susceptible de nuire à la cohésion sociale. Les récidivistes, de par la réitération continue de leurs forfaits, manifestent pour les initiateurs de la relégation un danger qu'ils font peser à l'ensemble de la société. La prison ne pouvant en venir à bout, il faut désormais adapter la pénalité en toute connaissance de cause, c'est-à-dire en prévenant les crimes et les délits qu'ils ne pourront manquer de commettre dans l'avenir. Ce faisant, c'est également autant de possibles révoltés ou 
de fomentateurs de révolte que la relégation soustraie du sol de la métropole pour les exiler perpétuellement sur le sol d'une colonie. Le traumatisme de la Commune résonne fortement dans le vote de cette loi et les récidivistes sont regardés comme de véritables ennemis de l'intérieur prêts à renverser une jeune République encore à ses débuts. Pour Pierre Waldeck-Rousseau, la relégation n'a d'autre but que de «préserver la société dans le présent et dans l'avenir en arrêtant le développement inquiétant de la criminalité6 ». La relégation comme la libération conditionnelle votée la même année obéissent ainsi à une logique de prévention et forme le chapitre d'une politique criminelle mise en place par les républicains opportunistes à partir de 1881 et qui repose en grande partie sur des conclusions dégagées par des experts du crime et des peines. Prévenir le crime, voilà le ressort de ce double dispositif contre la réci dive mis en place en 1885 puis complété en 1891 par la loi sur le sursis à exécution de la peine. Toutes deux s'attachent à éviter le recours à la prison et cherchent à prévenir le crime en luttant contre le récidivisme. La relégation est ainsi une loi de défense sociale censée s'assurer de récidivistes incorrigibles, c'est-à-dire insensibles à la pénalité classique et par là dangereux. Incapables de vivre au milieu d'une société trop avancée pour eux et de profiter de ses bienfaits, l'exil outremer permet de les frapper d'un ostracisme perpétuel et de les désolidariser d'une communauté nationale où ils n'ont pas leur place.

Jean-Lucien Sanchez

\section{Bibliographie :}

- Alline J.-P., Gouverner le crime. Les politiques criminelles françaises de la Révolution au XXIe siècle. 1. L'ordre des notables 1789-1920, Paris, L'Harmattan, 2003.

- Badinter R., La prison républicaine : 1871-1914, Paris, Librairie Générale Française, 1994.

- Beaud O., La puissance de l'État, Paris, PUF, 1994.

- Chauvaud F., «Repris de justice et incorrigibles : les figures du récidiviste au cœur de l'imaginaire judiciaire (France XIXe siècle) », Briegel F. et Porret M. (dir.), Le criminel endurci. Récidive et récidivistes du Moyen Age au XXe siècle, Genève, Droz, 2006.

- Chevalier L., Classes laborieuses et classes dangereuses, Paris, Perrin, 2002.

- Debuyst C., Digneffe F., Labadie J.-M. et Pires A., Histoire des savoirs sur le crime \& la peine, Paris, Bruxelles, De Boeck Université, 1996, 1998, 2 vol.

6 Waldeck-Rousseau P., Annales de la Chambre des députés, op. cit., séance du 11 novembre 1882, JO du 30 novembre 1882 , p. 81. 
- Ewald F., «La politique sociale des opportunistes, 1879-1885 », Berstein S. et Rudelle O., Le modèle républicain, Paris, PUF, 1992, p. 173-187.

- Farcy J.-C., «Le casier judiciaire au XIXème siècle », Bulletin du Centre d'Histoire de la France Contemporaine, $\mathrm{n}^{\circ} 11,1990$, p. 5-30.

- Fritz G., L’idée de peuple en France du XVIIème au XIXème siècle, Strasbourg, Presses Universitaires de Strasbourg, 1988.

- Garraud R., Traité théorique et pratique de droit pénal français, Paris, Larose et Forcel, 1888-1894, 5 vol.

- Jobert B., «La régulation politique : le point de vue d'un politiste », Commaille J. et Jobert B. (dir.), Droit et Société. Les métamorphoses de la régulation politique, Paris, LGDJ, 1998, p. 119-144.

- Kaluszynski M., La République à l'épreuve du crime. La construction du crime comme objet politique 1880-1920, Paris, LGDJ, 2002.

- Kaluszynski M., «La construction d’une politique « républicaine» de sécurité ? », Froment J.-C., Gleizal J.-J. et Kaluszynski M. (dir.), Les états à l'épreuve de la sécurité, Grenoble, Presses Universitaires de Grenoble, 2003, p. 15-30.

- Kaluszynski M., «Les artisans de la loi. Espaces juridico-politiques en France sous la IIIème République », Droit et Société. Produire la loi, Paris, LGDJ, 1998, p. 535562.

- Kaluszynski M., Production de la loi et genèse des politiques pénales. La Société générale des prisons 1877-1900, Grenoble, CERAT, 1996.

- Laborde C., «La citoyenneté », Duclerc V. et Prochasson C. (dir.), Dictionnaire critique de la République, Paris, Flammarion, 2002.

- Lacassagne A., «Marche de la criminalité en France de 1825 à 1880. Du criminel devant la science contemporaine », La revue scientifique de la France et de l'étranger, janvier à juillet $1881, \mathrm{t}$. I.

- Lascoumes P., Poncela P. et Lenoël P., Au nom de l'ordre. Une histoire politique du code pénal, Paris, Hachette, 1989.

- Leca J., «Individualisme et citoyenneté », Birnbaum P. et Leca J. (dir.), Sur l'individualisme, Paris, Presses de la Fondation Nationale des Sciences Politiques, 1986.

- Lochak D., «La citoyenneté : un concept juridique flou », Colas D., Emeri C. et Zylberger J. (dir.), Citoyenneté et nationalité : perspectives en France et au Québec, Paris, PUF, 1991, p. 179-207.

- Lombroso C., L’homme criminel : criminel-né, fou moral, épileptique : étude 
anthropologique et médico-légale, Paris, F. Alcan, 1887, 2 vol.

- Noiriel G., Les origines républicaines de Vichy, Paris, Hachette Littératures, 1999.

- Novel P., La récidive et le Code pénal, Paris, A. Rousseau, 1895.

- Perrot M., Robert P., Compte général de la justice criminelle en France pendant les années 1880 et rapport relatif aux années 1826 à 1880, Genève, Slatkin Reprints, 1989.

- Reinach J., La politique opportuniste, 1880-1889, Paris, Charpentier, 1890.

- Reinach J., Les lois de la République. Troisième législature, 1881-1885, Paris, Librairie Centrale des publications Populaires, 1885.

- Robert P., Le citoyen, le crime et l'Etat, Genève, Droz, 1999.

- Rosanvallon P., Le sacre du citoyen. Histoire du suffrage universel en France, Paris, Gallimard, 1992.

- Rusche G. et Kirchheimer O., Peine et structure sociale : histoire et théorie critique du régime pénal, Paris, Éditions du Cerf, 1994.

- Saada E., «Entre «assimilation» et «décivilisation». L'imitation et le projet colonial républicain », Terrain, $\mathrm{n}^{\circ} 44,2005$, p. 19-38.

- Saleilles R., L’individualisation de la peine : Étude de criminalité sociale, Paris, Alcan, 1927.

- Schnapper B., «La récidive, une obsession créatrice au XIXème siècle », Voies nouvelles en histoire du droit. La justice, la famille, la répression pénale (XVIème-XXème siècles), Paris, PUF, 1991, p. 313-351.

- Tanguy J.-F., « Ceux qu'il faut renoncer à amender... La loi de 1885 sur la relégation : origines et implications politiques », Briegel F. et Porret M. (dir), Le criminel endurci. Récidive et récidivistes du Moyen Age au XXème siècle, Genève, Droz, 2006.

- Wagniart J.-F., Le vagabond à la fin du XIXème siècle, Paris, Belin, 1999. 